\title{
SINGLE STAGE CORRECTION OF RECTOVESTIBULAR FISTULA
}

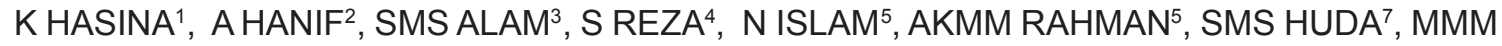 \\ PERVEZ ${ }^{8}$, SK MONDAL ${ }^{9}$, S ISLAM $^{10}$
}

\begin{abstract}
:
The aim of this study was to determine the feasibility, safety, cost effectiveness and functional outcome between single stage and multiple stage operation of anorectal malformation (ARM) with rectovestibular fistula (RVF).

Materials \& Methods: This interventional study was conducted on 40 female children born with ARM with RVF from July 2007 to June 2009 in the Department of Pediatric Surgery, Dhaka Medical College Hospital. Their age range was from 3 months to 7 years. Anterior sagittal anorectoplasty (ASARP) was done as definitive treatment of RVF. 20 out of $\mathbf{4 0}$ patients underwent single stage procedure and the rest underwent multiple stage procedure. Good preoperative bowel preparation and postoperative wound care were ensured for optimum outcome.
\end{abstract}

1. Dr. Kaniz Hasina, Assistant Professor, Department of Pediatric Surgery, Dhaka Medical College \& Hospital, Dhaka 1000, Bangladesh. Email: kanizhasina@gmail.com

2. Dr. Abdul Hanif, Assistant Professor, Department of Pediatric Surgery, Dhaka Medical College \& Hospital, Bangladesh.

3. Dr. S.M. Shafiqul Alam, Junior Consultant, Anesthesiology and ICU, Dhaka Medical College \& Hospital

4. Dr. Shahin Reza, Medical Officer, Upazilla Health Complex, Nawabgonj, Dhaka

5. Dr. Nazrul Islam, Medical Officer, Bangabandhu Shaikh Muzib Medical University (BSMMU)

6. Dr. A.K.M. Mizanur Rahman, Registrar, Dept. of Pediatric Surgery, Dhaka Medical College Hospital, Bangladesh.

7. Dr. S.M. Shamsul Huda, Post Graduate Training Fellow (FCPS-II), Bangabandhu Shaikh Muzib Medical University (BSMMU), Dhaka, Bangladesh.

8. Dr.M.M. Masud Pervez, $3^{\text {rd }}$ Part Student (MS), Department of Pediatric Surgery, Dhaka Medical College \& Hospital, Dhaka, Bangladesh

9. Dr. Susankar Kumar Mondal, Resident, Department of Pediatric Surgery, Dhaka Medical College \& Hospital, Dhaka, Bangladesh

10. Dr. Shahnoor Islam, Assistant Professor, Department of Pediatric Surgery, Dhaka Medical College \& Hospital, Dhaka

Correspondence to : Dr. Kaniz Hasina, Assistant Professor, Department of Pediatric Surgery, Dhaka Medical College \& Hospital, Dhaka 1000, Bangladesh, E-mail: kanizhasina @gmail.com
Result: Mean operating time was $\mathbf{7 0}$ minutes in single stage and 80 minutes in multi stage definitive procedure. Average hospital stay was 10 days in single stage procedure where as 22-24 days in multi stage procedure. Partial perineal wound dehiscence occurred in two patients with single stage procedure and one patient with multi stage procedure. Wound infection was seen in two patients of single stage and also two patients of multi stage procedures. Fecal continence was good in all patients older than 3 years. Average number of bowel movement was 2-3 times daily in older patients and 2-4 in younger patients. Constipation developed in three patients with multiple stage procedure and in two patients with single stage procedure. Both groups were managed conservatively for all complications. In Dhaka Medical College Hospital, treatment cost is mostly free, so we could not compare this cost but the incidental expenses in single stage treatment was Tk. 10,000.00 and Tk. 30,000.00 in multistage treatment in three settings.

Conclusion: Adequate preoperative bowel preparation, good surgical skill, proper analgesia and postoperative wound care remain the key factors to produce a cost effective excellent outcome of single stage correction of RVF.

Key Words: Rectovestibular Fistula (RVF), ASARP (Single Stage), Multiple Stage

Introduction:

Rectovestibular fistula (RVF) is the most frequent type of anorectal malformation (ARM) in female ${ }^{1}$. In these patients the rectum opens in the vestibule between the hymen and the posterior forchette ${ }^{2}$. The most important anatomic feature in this malformation to be kept in mind is that the rectum and the vagina share a common wall. The precise diagnosis is a clinical one and requires a meticulous inspection of the newborn genitalia. On clinical examination there appears a flat perineum with no anus and a third opening (the other two being the urethra and the vagina) in the introitus near the posterior forchette through which feces come out. 


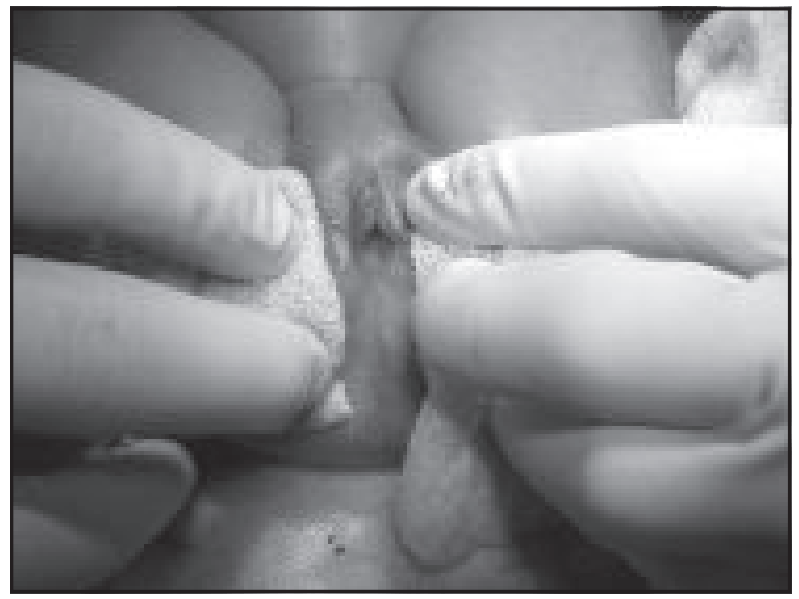

Fig.-1: ARM with RVF

Patients with RVF usually have well developed sphincter muscle complex at the perineum and a normal sacrum and nerves ${ }^{3}$. The presence of RVF can be diagnosed either by inserting a probe into the fistula and noting the direction, or by injecting contrast into the fistula ${ }^{2}$. Prognosis in terms of bowel function is usually good when treated properly. Despite a better understanding of embryology, anatomy and physiology of fecal continence, management of children born with RVF still remains a challenge to the pediatric surgeons due to numerous peroperative and post operative complications ${ }^{4-6}$.

Regarding management it is customary to create a diverting colostomy before the definitive surgery to protect the neoanus from fecal contamination ${ }^{7}$. But some surgeons like to correct the defect without a protective colostomy to reduce the number of operation from three (colostomy, anorectoplasty and closure of colostomy) to one (definitive procedure i.e. anorectoplasty) as well as to avoid the complications and adverse psychological effect of a colostomy ${ }^{7-9}$. Usefulness of any surgical procedure for ARM is based on its future bowel function and continence. Patients with RVF born with good bowel control. So effort should be made to give them the best opportunity to have a successful reconstruction as a single stage procedure $^{10}$.

\section{Materials and Methods:}

This was a prospective study done over a period of 2 years from July 2007 to June 2009 and carried out in the Department of Pediatric Surgery, Dhaka Medical College and Hospital, Dhaka. Total 2218 patients were

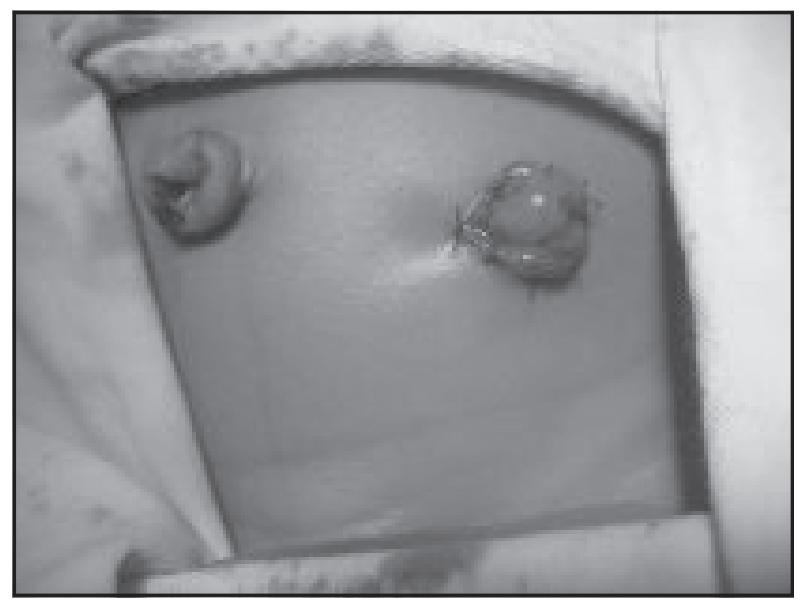

Fig.-2: RVF with pelvic colostomy

admitted, of which 158 were patients with ARM . About 55 girls were selected initially with vestibular fistula, 15 were anovestibular fistula (AVF) excluded from the study and 40 patients with RVF were included in this study by purposive sampling. Their age range was from 3 months to 7 years. Anterior sagittal anorectoplasty (ASARP) was done as definitive treatment. 20 out of 40 patients underwent primary reconstruction without colostomy (i.e. single stage procedure) and the rest underwent multiple stage procedure (colostomy, definitive surgery and closure of colostomy). Routine investigations like $\mathrm{Hb} \%$, total and differential count of WBC, serum electrolytes, serum creatinine, blood grouping and cross matching, ultrasonogram of kidneys, ureters, bladder (KUB) and pelvic organs and X-ray chest were done.

Preoperative bowel preparation was ensured by three days distal loop and fistula irrigation (using normal saline, liquid paraffin and glycerin) in presence of protective colostomy. Oral antibiotics (Ciprofloxacin $30 \mathrm{mg} / \mathrm{kg} /$ day divided 12 hourly and Metronidazole $20 \mathrm{mg} / / \mathrm{kg} /$ day divided 8 hourly) for 2 days and intravenous Ciprofloxacin 12 hourly in above mentioned dose and Metronidazol $1.5 \mathrm{ml} / \mathrm{kg} /$ dose 8 hourly from the day before surgery were started and the patients were kept nothing per oral (NPO) for 6 hours preoperatively. In single stage, fistula irrigation was started with the same preparation from three days prior to surgery. The patients were kept only on plain water and ORS orally with oral antibiotics for two days and NPO, intravenous infusions with antibiotics (Ciprofloxacin and Metronidazole) from day before surgery. In addition, injection Mannitol $(0.5-1 \mathrm{gm} / \mathrm{kg} /$ 
dose, 12 hourly) was given per oral on the day before surgery.

The operation was performed under general anesthesia along with caudal block. During operation a catheter was inserted in the urinary bladder to protect the urethra as well as the neoanus postoperatively from urinary contamination. Preoperative calculative dose of Ceftriaxone, $100 \mathrm{mg} / \mathrm{kg}$ and Metronidazole, $1.5 \mathrm{ml} /$ $\mathrm{kg}$ were given intravenously after induction of anesthesia.

Most critical part of the operation is the dissection of the anterior rectal wall which also forms the posterior wall of vagina. As tissue volume is small, careful and meticulous dissection in the perineum is also required. It needs very delicate surgery to create a plane to make two walls out of one. Mobilization of the rectum is done upto the point where rectum and vagina bear separate walls. Incomplete separation of rectal and vaginal wall may create a tense anastomosis between the rectum

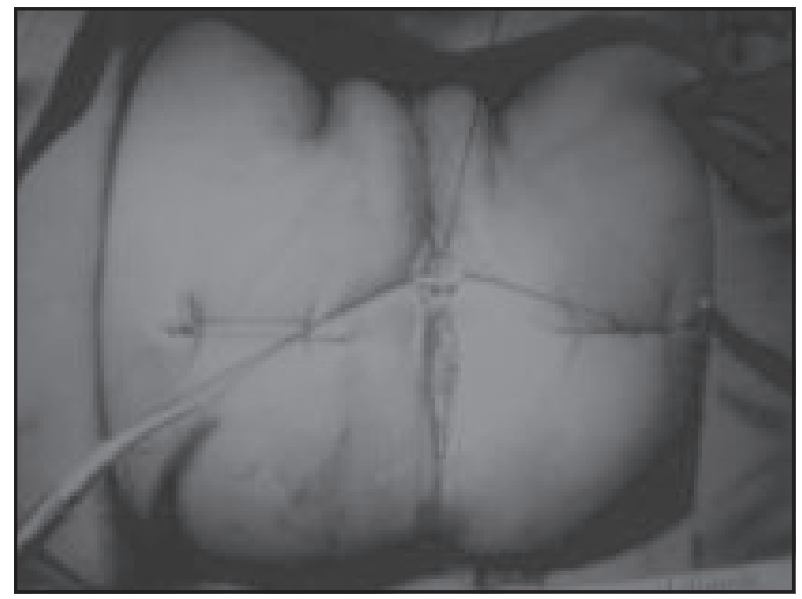

Fig. 3: Racket incision of ASARP

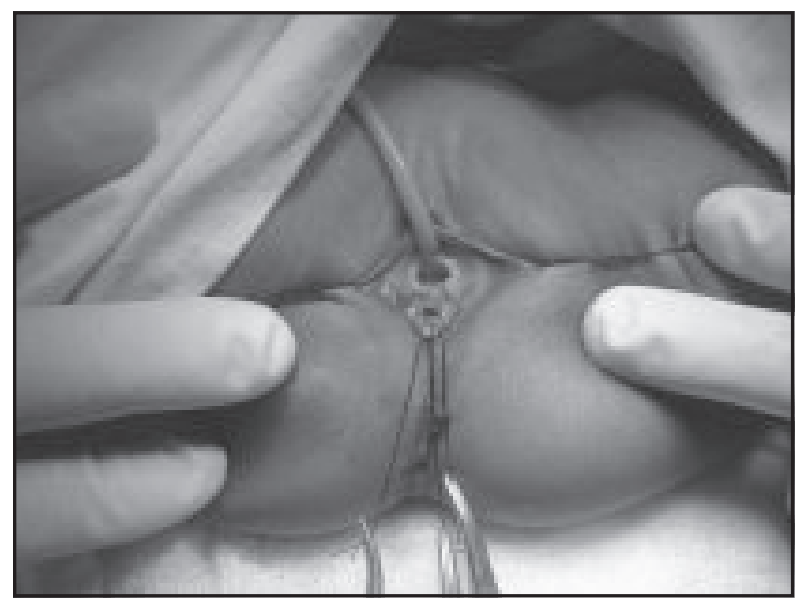

Fig. 4: Rectum is separating from vagina and perineal skin. This may provoke wound dehiscence and recurrence of fistula. Proposed anal site was determined by the anal mark and confirmed by the use of an electro stimulator. After preparing and draping, a circular incision was made in the mucocutaneous junction at the opening of the fistula and extended posteriorly along the midline to reach the anal dimple (so called racket incision). Several fine silk traction sutures were inserted around fistula orifice.

Through the incision, anorectum was dissected bluntly from the surrounding musculature enclosing the rectum and the connective tissues between rectum and vagina upto the point where rectum and vagina bear separate wall. Then mobilized rectum was placed at the centre of the fan shaped muscles. Anterior end of the fan shaped muscle fibres were apposed and sutured by interrupted sutures with $4-0$ vicryl. The rectum was fixed to the deep external sphincter muscles by $4-0$ vicryl. Anoplasty was done by about 12-16 stitches with 4-0 vicryl. Vestibular wound was

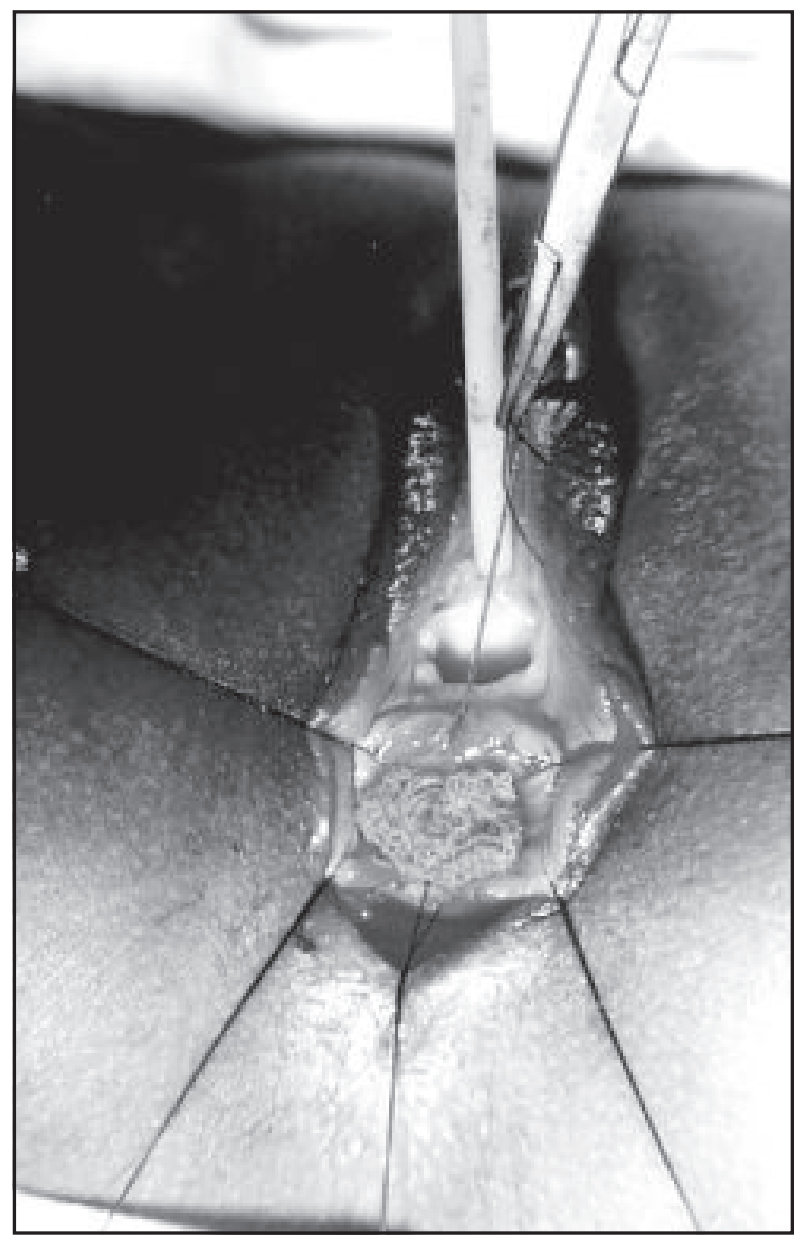

Fig. 5: RVF during surgery 


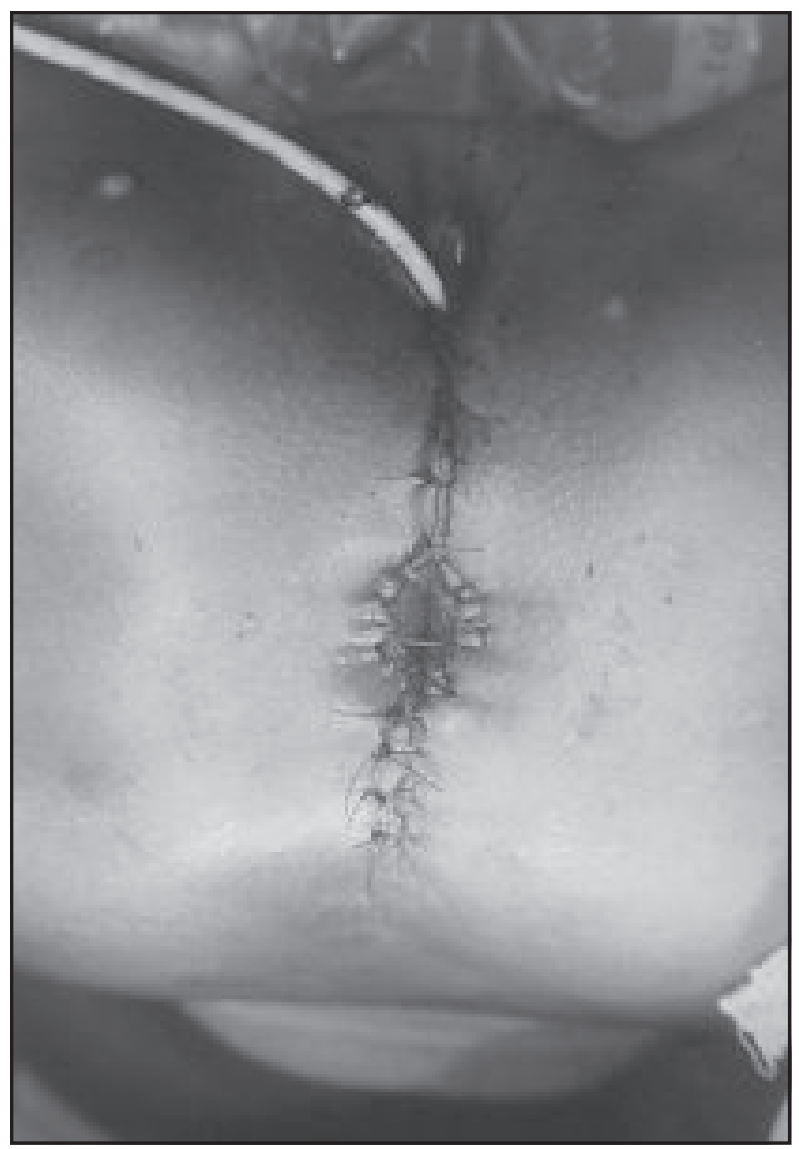

Fig. 6: Neoanus after surgery

closed with interrupted stitches and vaginal introitus was also fixed to the posterior forchette.

Postoperatively patients were kept nothing per oral for four days in single stage and one day or till colostomy was functioning in multistage procedure. Intravenous antibiotics (ceftriaxone, metronidazol and gentamicin, $2 \mathrm{mg} / \mathrm{kg} /$ day 8 hourly) continued for five days along with eight hourly pethidine $(1-1.5 \mathrm{mg} / \mathrm{kg} /$ dose) for 2 days in single stage and 1 day in multistage. Rectal suppository was strictly forbidden. Suppositories (paracetamol or diclofenac sodium depending on the age of the patients, upto six months paracetamol and after six months, diclofenac sodium) were used through distal loop in case of multi stage procedure. Perineal cleanliness was maintained by spraying the neoanus by povidone iodine mixed normal saline as well as local application of povidone iodine cream for five days.

Catheter was removed on the $5^{\text {th }}$ postoperative day (POD). Majority of the patients were discharged on the $7^{\text {th }}$ POD of surgery unless indicated for some reason. Anal dilatation was started on $14^{\text {th }}$ POD. Parents were informed about the planned schedule of follow up and anal dilatation. The multistage patients were advised to come after completion of anal dilatation for colostomy closure.

The patients were advised to visit the hospital two weeks after surgery. The anus was calibrated with metallic anal dilators (calibrated with the Hegar dilators of appropriate size) that fitted snuggly in the anus. The dilator was given to the mother and instructed her to dilate the neoanus twice daily. Every weekly the size of the dilator must be changed to the next size one. The parents were encouraged to do it by themselves at home as advised. Once the desired size dilator has reached with no pain (usually 3-4 weeks after the last size dilator was reached), one may start tapering the frequency of the dilatation as follows ${ }^{10}$ :

Once a day for a month.

Every other day for a month.

Every third day for a month.

Twice a week for a month.

Once a week for a month.

Once a month for three months.

Optimal dilator size (Kiely and Pena,1998):

\begin{tabular}{lc}
\hline Patient age & Hegar dilator \\
\hline 1-4 months & 12 \\
$4-8$ months & 13 \\
8-12 months & 14 \\
1year-3 years & 15 \\
3 years-12 years & 16 \\
> 12 years & 17 \\
\hline
\end{tabular}

\section{Result:}

40 patients having ARM with RVF were included in the study. 20 patients were treated by single stage i.e. primary anorectoplasty (ASARP). Other 20 were underwent multistage treatment i.e. initial defunctioning sigmoid colostomy followed by anorectoplasty (ASARP); in the third stage the colostomy was closed to maintain bowel continuation. Mean operating time was 70 minutes for single stage procedure and total 220 minutes ( $50 \mathrm{~min}$. for colostomy +80 min for anorectoplasty +90 min for colostomy 
closure) multistage treatment. Average hospital stay was 10 days in single stage and 22-24 days $(5+11+6$ to 8 ) in multistage management.

Surgical outcome was assessed during postoperative period and follow up visits. In single stage treatment tearing of posterior vaginal wall occurred in one patient during separation of common wall between rectum and vagina. Similar injury occurred in two patients in multistage treatment. All the patients were examined on $2^{\text {nd }}$ and $5^{\text {th }}$ POD to see the presence or absence of wound infection and wound dehiscence. Partial perineal wound dehiscence occurred in two patients with single stage procedure and one patient with multi stage procedure. Wound infection was also seen in two patients of single stage and two patients of multi stage procedures. After final procedure, constipation developed in two patients with single stage treatment and three patients with multi stage treatment. All the five patients were managed conservatively (diet, drug and bowel training). The patients were followed up according to the schedule of the anal dilatation protocol planned initially. They visited the hospital at 14 days of surgery ( $1^{\text {st }}$ follow up), 1 month after $1^{\text {st }}$ follow up. 1 month after $2^{\text {nd }}$ follow up and 1 month after 3rd follow up for colostomy closure of multistage procedure. During each visit, appearance and size of the neoanus and presence or absence of any complications (wound infection, wound disruption, mucosal prolapse and fistula formation) were noted.

As the Hegar dilator set is very expensive, the anal dilatation at home was carried out by local made metallic anal dilators, which were calibrated with the Hegar dilators of appropriate size at operation theatre at each visit by the researcher himself/herself. The follow up schedule was three months in single stage and more in multi stage upto colostomy closure.

Functional outcome (that is continence) of the operation in children older than 3 years was good. Average number of bowel movement was two to three times daily in these patients several weeks postoperatively.

\section{Discussion:}

In 24 months study, total 2218 patients were admitted in the Pediatric Surgery Department of Dhaka Medical College Hospital, of which 158 (7.12\%) were patients with ARM . About 55 (34.81\%) girls were selected initially with vestibular fistula. Among them, 15 (9.49\%) were clinically diagnosed as anovestibular fistula and
40 (25.32\%) patients as RVF by probing the fistulous tract and observing the direction. In this study, RVF was larger in number than anovestibular fistula. This observation is similar to Pena (2003b) and Freeman (1994). Islam (1992) also found RVF as frequent female ARM defect ${ }^{11}$.

All 40 patients of RVF presented with absence of anal opening, passage of meconium through vestibular fistula.

The operative procedures performed in this study was ASARP with or without colostomy. In single stage procedure, out of 20 patients 01 (5\%) patient had vaginal wall injury and in multiple stage, $02(10 \%)$ patients had vaginal wall injury among 20 patients. Those vaginal tear were repaired during operation, none of them developed further complication i.e. fistula formation.

Minimum time required for definitive surgery in single stage was $70 \mathrm{~min}$ and total time required for multiple stage procedure was $220 \mathrm{~min}$ (in three stage). Hospital stay in single stage was about 10 days and 22-24 days in multi stage.

All the patients were examined on $2^{\text {nd }}$ and $5^{\text {th }} P O D$ to see whether wound infection and wound dehiscence were present or not. Partial perineal wound dehiscence occurred in $02(10 \%)$ patients with single stage procedure and $01(5 \%)$ patient with multi stage procedure. Wound infection was also seen in $02(10 \%)$ patients of single stage and $02(10 \%)$ patients of multi stage procedures. After final procedure constipation developed in $02(10 \%)$ patients with single stage treatment and $03(15 \%)$ patients with multi stage treatment.

Being the largest public hospital of Bangladesh, in Dhaka Medical College Hospital treatment cost is mostly free, so we could not compare this cost but incidental expenses in single stage treatment was Tk. 10,000.00 and Tk. 30,000.00 in multistage treatment.

Principal advantages of primary anorectoplasty without a defunctioning colostomy are avoidance of multiple operations and complications of a colostomy. An efficacious and cost effective care of patients of ARM with RVF begins with a carefully thought out plan in neonatal period. The traditional surgical correction entails a diverting colostomy, usually in the neonatal period, anorectoplasty at about one year and finally closure of colostomy several months later. 
Diverting colostomy in a baby with RVF is mostly unnecessary as the gastrointestinal tract can decompress itself through the fistula. Patients' and parents' distress as well as complications of a colostomy is well known to all surgeons. Moreover added financial burden of a multistage treatment, especially in a third world country, has also been highlighted in this article.

A large number of patients fail to come for follow up in the postoperative months after a colostomy. Background causes are ignorance, lack of awareness, poverty, family problems and distance of a tertiary hospital from a remote village.

Advantages of avoiding a colostomy are many. Colostomy related complications are completely eliminated. This is even more important in developing countries where a colostomy is socially unacceptable, trained stoma nurses and pediatric colostomy appliances are mostly unavailable, and parents are ignorant and not conscious about proper care of a colostomy.

A multistage treatment of RVF means three admissions separating the mother and possibly the father from rest of their children. Cost of long hospital stay as well as three surgeries is definitely a burden to the most of the parents. Occasionally it exceeds the annual income of the family. It is, therefore, be definite an advantage if the patient can have only one admission, no colostomy and the parents have to pay for single operation. In this study definitive surgery was deferred for three months in neonates presented with ARM with RVF to avoid anesthetic hazards and inadequate facilities of neonatal high dependency care in our settings. In this period intestinal obstruction is uncommon as stool of breast fed babies is liquid to semi liquid. Moreover irrigation through the fistula was given whenever required.

Fecal contamination of the perineal wound was avoided by preoperative gut preparation, postoperative abstinence from oral feeding, intravenous antibiotics and application of local antiseptic.

Vaginal tear occurred in one patient $(5 \%)$, partial perineal wound dehiscence in two $(10 \%)$ and wound infection in two patients (10\%) who were treated by primary anorectoplasty without colostomy. This incidence of complication is not higher than those of multistage management. Average hospital stay was 10 days in single stage and 22-24 days in multi stage procedures. Mean operation time was 70 minutes for single stage procedure and total 220 minutes for multistage treatment. Operation and related cost was almost three times more in multistage than in single stage treatment.

\section{Conclusion:}

Results of this study on single treatment, i.e. primary anorectoplasty, of RVF is definitely encouraging regarding patient and parental compliance, cost benefit and surgical outcome. Good preoperative gut preparation, meticulous surgical technique and good postoperative care are the key factors for a successful single stage primary reconstruction of rectovestibular fistula. In our perspective, though the complication rate in both the procedures were very close to each other, single stage correction of RVF is more acceptable than multi stage correction.

\section{References :}

1. Wakhlu A.,Pandey A., Prasad A., Kureel S.N., Tandon R.K., and Wakhlu A.K., 1996, "Anterior sagittal anorectoplasty for anorectal malformations and Perineal trauma in the female child" , J. Pediatr Surg, vol.31, no. 9, pp. 12361240.

2. Freeman N.V. 1994, "Anorectal malformations", in surgery of the Newborn, eds. , D.M. Burge, M. Griffiths and P.S.J. Maline, Churchill Livingstone, Edinburgh, pp. 171-199.

3. Pena A. ,2003b, "Anorectal anomalies" in Newborn Surgery, $2^{\text {nd }}$ edn, eds. P.Puri, Arnold, London, pp. 535-552.

4. Smith E.D. 1987, "The bath water needs changing, but donot throw out the baby : An overview of anorectal anomalies", J. Pediatr Surg, vol.22, no. 4, pp. 335-388.

5. Yazbeck S. , Luks F.I., and St-Vil D. , 1992, "Anterior perineal approach and three flap anorectoplasty for imperforate anus : Optimal reconstruction with minimal destruction", J. Pediatr Surg, vol.27, no. 2, pp. 190-195.

6. Sigalet D.L., Laberge J.M., Adolph V.R., and Guttman F.R., 1996, "The anterior sagittal approach for high imperforate anus: A simplification of the Mollard approach", J. Pediatr Surg, vol.31, no. 5, pp. 625-629.

7. Pena A. 2000," Imperforate anus and cloacal malformations" in pediatric surgery, $3^{\text {rd }}$ edn, eds. K.W. Ashcraft, W.B. Saunders company, Philadelphia, pp. 473-492. 
8. Moore T.C., 1990, "Advantages of performing the sagittal anoplasty operation for imperforate anus at birth", J. Pediatr Surg, vol.25, no. 2, pp. 276277.

9. Okada A., Shinkichi K., Imura K., Fukuzawa M., Kubota A., Yagi M., Azuma T., and Tsuji H., 1992, "Anterior sagittal Anorectoplasty for Rectovestibular and Anovestibular fistula", J. Pediatr Surg, vol.27, no. 1, pp. 85-88.
10. Stephens F.D., And Smith D., 1986, "Classification, identification, and assessment of surgical treatment of anorectal anomalies", Pediatr Surg Int., vol.1, pp. 200-205.

11. Islam, M.K. 1992, 'Pattern and etiology of anorectal anomaly', M.S. thesis, University of Dhaka, Dhaka. 